\title{
Ernst Nolte
}

\section{Zwischen Totalitarismus und bürgerlicher Gesellschaft: Fehlentwicklungen des internationalen Systems im 20. Jahrhundert}

Das Thema bedarf der Erläuterung und Präzisierung. Unter dem „internationalen System“ ist offenbar das „internationale Staatensystem“ zu verstehen, und vom Anfang des 20. Jahrhunderts an wurde mehr und mehr von einem „Weltstaatensystem" gesprochen. Insofern ist die Fragestellung in die Nachfolge von Rankes "großen Mächten“ zu stellen, und in der Gegenwart mag als bekanntes Beispiel Paul Kennedys „Aufstieg und Fall der großen Mächte“ herangezogen werden. Diese Betrachtungsweise ist das eigenste Eigentum der Geschichtswissenchaft; kein anderes Fach hat hier ernsthaft mitzureden. Ihrem Idealtyp nach handelt es sich um eine streng realistische Betrachtungsweise, die sich moralische Urteile verbietet; der Aufstieg Spaniens im 16. Jahrhundert wird ebenso ohne Parteinahme und tadelnde Urteile dargestellt wie der Niedergang Österreich-Ungarns seit der Bildung des italienischen und des deutschen Nationalstaates; worauf es ankommt, ist das Machtpotential der einzelnen Staaten, das zu einem wesentlichen Teil auf der Produktivkraft von Landwirtschaft, Industrie und Handel, aber auch auf der Effizienz oder Ineffizienz der politischen Organisation beruht und das jedem Staat seinen beherrschenden oder untergeordneten Platz in dem internationalen System anweist. Die Verschiebungen dieser Machtbalance der großen Mächte zu verfolgen, ist die höchste Aufgabe der Geschichtswissenschaft. Sie darf sich daher nicht scheuen, etwa ohne tadelnden oder verwunderten Unterton festzustellen, daß Deutschland im Jahre 1917 dicht vor dem endgültigen Siege gestanden habe oder durch Statistiken deutlich zu machen, daß der Jahresindex der Industrieproduktion 1938 im Falle Deutschlands und auch der USA das Anderthalbfache des Index von 1913 betragen habe, im Falle der Sowjetunion aber das Achtfache ${ }^{1}$. Allenfalls der Ansatz eines moralischen Urteils kann, wie bei Kennedy, durch den Begriff der „Überdehnung“ hineingebracht werden, und nur

1 Paul Kennedy, Aufstieg und Fall der großen Mächte. Ökonomischer Wandel und militärischer Konflikt von 1500 bis 2000 (Frankfurt a.M. 1989, Orig.ausg. New York 1987) 408, 451. 
wenn eine Gesamtvorstellung vom Scheitern aller Hegemonialbestrebungen in Europa vorliegt, wie bei Ludwig Dehio, kann der Versuch Hitlers, die Herrschaft über den Kontinent zu gewinnen, mit moralischem Akzent verurteilt werden.

Aber im Titel wird auch der Begriff der "bürgerlichen Gesellschaft" verwendet. Darunter ist offensichtlich zunächst das innenpolitische System einiger der in Frage stehenden Mächte zu verstehen, ein System, das häufig „westliche Demokratie" genannt wird, das man aber besser als Liberales System bezeichnen sollte, denn um 1910 konnte weder in Deutschland noch in England ernsthaft von „Demokratie" die Rede sein. Wohl aber gab es Artikulations- und Organisationsfreiheit für alle möglichen politischen Tendenzen einschließlich einer radikalen und systemfeindlichen Opposition, es gab also Parteien, Parlamente und Wahlen, und die Freiheit des Geisteslebens war so gut wie unumschränkt. Das Wirtschaftsleben war durch ein hohes Maß an freier Konkurrenz zwischen Individuen und Firmen gekennzeichnet, und das Streben nach Autarkic war weit weniger ausgeprägt als die Tendenz zur Erweiterung des Welthandels. Die Anfänge dieses „freiheitlichen Systems" waren schon seit 1815 trotz aller Restaurationen und Reaktionen klar erkennbar, und man mochte sie sogar auf mittelalterliche Wurzeln zurückführen. Alle großen Mächte des Weltstaatensystems waren zugleich Bestandteile eines internationalen Liberalen Systems, das eine Anzahl von Regeln anerkannte und das sich durchweg als den fortgeschrittenen Teil der Menschheit betrachtete. Ein optimistischer Blick in die Zukunft war vorherrschend: die Fortschritte der Naturwissenschaft und des vernünftigen Denkens würden in absehbarer Zeit auch die zurückgebliebenen Teile der Welt auf die Stufe der Modernität heben, und vielleicht würde sogar ein rationaler Interessenausgleich an die Stelle der kriegerischen Machtkämpfe treten. Aber die Verfechter des Pazifismus und des Kosmopolitismus bildeten doch nur eine Art Vorhut, und Rußland sowie Japan müßte man als eine Nachhut ansehen, die noch einige Zeit benötigen würden, bis auch sie „europäisiert“ sein würden. Es gab um 1910 nichts, was diesem „Liberalen System" gleichgewichtig gegenübergestanden hätte, und ein Regime wie der Bonapartismus Napoleons III. war nur noch eine ferne Erinnerung.

Aber seit 1917 kam ein Regime zur Existenz, auf das ein deutscher Schriftsteller, Alfons Paquet, aus unmittelbarer Anschauung heraus schon im Sommer 1918 den Terminus „revolutionärer Totalismus" anwandte ${ }^{2}$. Zwar ging die russische Oktoberrevolution direkt aus der Niederlage des Zarenreiches im Krieg gegen Deutschland hervor, und ohne das verzweifelte Aufbegehren der Soldatenmassen gegen die unerträglichen Lasten des bereits verlorenen Krieges hätte sie schwerlich stattgefunden, aber sie schloß doch zugleich die tiefsten Überzeugungen der sozialistischen Bewegung Europas in sich und betrachtete sich selbst als das exakte Gegenteil jener Konzeption vom unaufhörlichen Ringen der großen Mächte, das sie als "Imperialismus" verwarf und mit dem unmenschlichen Ausbeutungssystem des modernen, wenn auch bislang fortschrittlichen Kapitalismus zusam-

2 Alfons Paquet, Im Kommunistischen Rußland. Briefe aus Moskau (Jena 1919) 111. 
menbrachte. Eine neue und weit machtvollere Großmacht würde alle bisherigen Großmächte und deren System hinwegfegen, nämlich die Großmacht der Weltrevolution, welche zur Wirklichkeit machen würde, was innerhalb des Liberalen oder kapitalistischen Systems nur von einer Minderheit fortschrittlicher Menschen erträumt worden war: die einheitliche Menschheit ohne Klassen, Staaten, Konkurrenz, Ausbeutung und Herrschaftsapparate. Was in der Verschwörung Babeufs und im Aufstand der Pariser Kommune nicht zur Entfaltung gelangt war, erzielte hier den entscheidenden Durchbruch: die „Ewige Linke“ in Gestalt des Marxismus. Wer sich die Gläubigkeit, den Schwung und den Enthusiasmus der frühen Verlautbarungen der Kommunistischen Internationale vor Augen stellt, der kann nicht umhin einzuräumen, daß hier etwas schlechthin Außerordentliches zur Wirklichkeit geworden war, das sich ebensosehr der bürgerlichen Gesellschaft wie der Konzeption von den "großen Mächten“, mit einem Wort: dem Liberalen System, grundsätzlich entgegenstellte und das den Terminus „Fehlentwicklung“ voller Verachtung zurückgewiesen hätte.

Aber die russische Revolution war auch ein Ausbruch elementarer Wildheit und Volksleidenschaft, die ebensosehr ein schreckenerregendes wie ein enthusiasmierendes Gesicht hatte, und erstmals in der europäischen Geschichte vollzog sich eine genuine und umfassende Klassenvernichtung, welcher der Adel, das Bürgertum und dann auch das selbstwirtschaftende Bauerntum zum Opfer fielen. Nicht zufällig drängten sich daher schon früh vielen Beobachtern Termini wie „asiatisch“ oder mindestens „die neuen Zaren“ über die Lippen. Spätestens mit dem Scheitern des „deutschen Oktober“ 1923 und dem Tode Lenins zerstoben die Hoffnungen auf die bevorstehende Weltrevolution und damit auch auf die Befreiung der russischen Avantgarde aus ihrer Fesselung an zurückgebliebene Verhältnisse; eine neue Normalität schien sich mit der Herrschaft des Georgiers Stalin abzuzeichnen; Sowjetrußland, jetzt „Union der Sozialistischen Sowjetrepubliken“ genannt, schien wieder in das neue System der Großmächte eingetreten zu sein, in das System des Versailler Friedens, wenn auch als eine marginale und geschwächte Großmacht. Aber das marxistische Verständnis der Gegenwart blieb gleichwohl dem liberalen oder evolutionär-optimistischen oder der Völkerbundsidee entgegengesetzt: Für Stalin und die KPdSU war die Sowjetunion nach wie vor der revolutionäre Staat schlechthin, der in aller Welt und zumal in Deutschland zahllose Anhänger besaß, der die Hoffnungen der Kolonialvölker auf sich zog und der ab 1928 ein präzedenzloses Unternehmen industriellen Aufbaus sowie militärischer Aufrüstung ins Werk setzte. Und auch die orthodox-marxistische Auffassung der Kautsky und Otto Bauer wurde mit der liberalen nicht identisch, denn sie suchte die Sowjetunion als eine Entwicklungsdiktatur zu begreifen, der zwar nur eine begrenzte, aber doch eine positive Rolle in der künftigen Weltentwicklung zuzuschreiben sei.

Eine spezifische Antwort entwickelte die liberale Auffassung erst, als sich seit dem Jahre 1922 in Italien ein Regime durchsetzte, das schroff antikommunistisch war und dennoch sehr merkwürdige Ähnlichkeiten mit dem sowjetischen Regime aufwies: die Monokratie einer Staatspartei und ihres Führers, die straffe Lenkung 
des geistigen Lebens, die Ausschaltung aller politischen Gegner, die Propagierung einer einzigen Ideologie usw. Jetzt gelangte die Lehre vom Totalitarismus zur Ausbildung, zunächst in Italien, und sie gewann an Bedeutung, als im Jahr 1933 Hitler und die NSDAP in Deutschland zur Macht kamen. Aber stärker und einflußreicher waren noch für mehrere Jahre die überwiegend marxistischen „Theorien über den Faschismus", die einen kausalen Nexus zwischen dem Imperialismus als bisher letztem Stadium des westlichen Kapitalismus und dem Faschismus herstellten, so daß die Sowjetunion immerhin als "antifaschistische Macht" angesehen werden konnte. Die intellektuelle und die politische Auseinandersetzung der dreißiger Jahre spielte sich also nicht so sehr zwischen "Totalitarismus" und "bürgerlicher Gesellschaft bzw. Totalitarismuskonzeption“ ab als vielmehr zwischen philosowjetischem Antifaschismus und philofaschistischem Antikommunismus. Selbst Paul Kennedy kommt nicht umhin, den Begriff „faschistische Mächte" zu verwenden, der ein ideologisches Moment in die prinzipiell unideologische und unmoralische (oder übermoralische) Konzeption der "großen Mächte“ einführt, und ein Buch wie dasjenige von David Davies, das 1930 ganz im Geiste der noch optimistischen Völkerbundsidee die Etablierung einer internationalen Polizeimacht gefordert hatte, schien 1936 einer fernen Vorzeit anzugehören ${ }^{3}$. Winston Churchill, cinst der mächtigste und entschlossenste Feind des Kommunismus' und Sowjetrußlands, verlangte jetzt ein Bündnis der Westmächte und der Sowjetunion zwecks Widerstands gegen die aggressiven und revisionistischen Ansprüche Hitler-Deutschlands, und Neville Chamberlain neigte allem Anschein nach zu einem antisowjetischen Ausgleich mit dem Deutschen Reich. Daneben hielt sich auch in England und Amerika die Auffassung, im weltpolitischen Spiel der Mächte handle es sich wesentlich um einen Kampf der „Have-nots“ wie Deutschland, Italien und Japan gegen die „Haves“ wie Großbritannien, USA und auch UdSSR, der durch ein Entgegenkommen der besitzenden Länder entschärft werden sollte; so etwa in dem Buch von Frank H. Simonds und Brooks Emeny "The Great Powers in World Politics“ von 19394. Nur eine marginale Rolle spielte die älteste aller Geschichtskonzeptionen, die ursprünglich katholische Verfallslehre von der fortschreitenden Säkularisierung, welche mit Begriffen wie „Revolution des Nihilismus" zu ganz pessimistischen Ausblicken gelangte. Erst der Hitler-Stalin-Pakt schien 1939 die Entgegensetzung von „Totalitarismus“ und „bürgerlicher Gesellschaft“ bzw. „Liberalem System“ ganz in den Vordergrund zu bringen, und die Kampfschrift des ehemaligen Kommunisten Franz Borkenau „The Totalitarian Enemy“ war 1939 so etwas wie ein Trompetenstoß. Die Pläne der Engländer und Franzosen, durch Luftangriffe gegen Baku und andere Aktionen in den Angriffskrieg der Sowjetunion gegen Finnland einzugreifen, würden eine definitive politische Realisierung dieser Konzeption bedeutet haben, doch

${ }^{3}$ David Davies, The Problem of the XXth Century. A Study in International Relationships (London 1930).

${ }^{4}$ Frank H. Simonds, Brooks Emeny, The Great Powers in World Politics (New York 1939). 
Hitlers Angriff gegen die Sowjetunion ließ abermals die antifaschistische und nun nicht mehr exklusiv-kommunistische Konzeption vom Kampf der demokratischen Völker gegen die faschistischen „Feinde der Menschheit" die Oberhand gewinnen. Aber nach dem Triumph von 1945 gelangte die elementare Feindschaft zwischen "Kapitalismus" und "Kommunismus", zwischen "freier Welt" und "Totalitarismus" rasch von neuem ins Offene, und erst jetzt wurde auf westlicher Seite die „klassische Totalitarismustheorie“ in Gestalt der großen Werke von Hannah Arendt und Friedrich/Brzezinski entwickelt. Im „Kalten Krieg“ trat für die USA die Sowjetunion als Hauptfeind an die Stelle des nationalsozialistischen Deutschland, und für die Sowjetunion stellten sich die Vereinigten Staaten als die direkten und noch gefährlicheren Nachfolger Hitlers dar. In der Perspektive der Lehre von den „Großen Mächten“ lag nichts anderes als der Kampf von zwei „Supermächten " um dic Herrschaft über die zunächst noch bipolare Welt vor, und in dicsem Kampf waren Ideologien und Konzeptionen untergeordnete, wenngleich nicht unwichtige Kampfmittel. Es mochte sich wie ein für die USA unheilvolles Vorzeichen ausnehmen, daß in den sechziger und siebziger Jahren die Totalitarismustheorie in großen Teilen der westlichen Welt zurückgedrängt, ja heftig bekämpft wurde, und zwar zugunsten neuer und bald überwiegend neomarxistischer „Theorien über den Faschismus“, die mit dem schroffen Antiamerikanismus der weltweiten Empörung über den imperialistischen „Vietnam-Krieg“ meist aufs beste zusammenstimmten. Aber im Rückblick aus der Zeit nach der Entscheidung von 1989/91, d. h. der Wiedervereinigung Deutschlands, dem Zusammenbruch der kommunistischen Regime in Osteuropa und der Auflösung der Sowjetunion in ihre Bcstandteile, läßt sich vermutlich sagen, daß das Nebeneinander von scheinbar oder wirklich philosowjetischer Entspannungspolitik und partiellem Festhalten an Elementen der Totalitarismuskonzeption nichts anderes als eine objektive „Doppelstrategie“ des Liberalen Systems bzw. der westlichen Demokratie war, um jenes inzwischen erstarrte „Außerordentliche“, das 1917 in die Welt gekommen war, auf andere Art zu besiegen, als Adolf Hitler es mit seiner direkten und schließlich militärischen Entgegensetzung versucht hatte. Im Jahre 1991 ging die „Ära des Kalten Krieges“ zu Ende, welche seit 1945/47 die „Epoche des Faschismus" abgelöst hatte, aber ebenso wie diese zu dem Zeitalter gerechnet werden muß, das nach dem Namen der stärksten Herausforderung der vorwiegenden Norm oder des „Normalen“ innerhalb des Jahrhunderts, das Zeitalter des chiliastischen Gewaltkommunismus genannt werden sollte. $\mathrm{Ob}$ ihm ein „Ende der Geschichte" folgen wird, d. h. ein Weltalter der unbestrittenen Herrschaft der liberalen, auf Verhandlungen und Kompromisse ausgerichteten Demokratie, wie Francis Fukuyama behauptete, oder ob ein Ringen von Zivilisationen an die Stelle des Kampfes der großen Mächte treten wird, wie Samuel P. Huntington glaubt, oder ob die Welt im Chaos ethnischer und sozialer Konflikte versinken wird oder ob gar am Ende eine Öko-Diktatur als rettender Totalitarismus die Menschheit vor der Selbstvernichtung bewahren wird, darüber könnte am Ende des 20. Jahrhunderts nur ein Prophet, aber sicherlich kein Historiker etwas Überzeugendes sagen. 
Trotz aller gebotenen Vorsicht ist indessen die Behauptung zulässig, am Ende des 20. Jahrhunderts habe das Liberale System oder, wenn man will, die pluralistische Demokratie - einen spektakulären Sieg errungen, und das gleiche müsse von der Totalitarismuskonzeption gesagt werden, die seine adäquateste intellektuelle Ausdrucksform sei. Ich will daher abschließend noch einige Bemerkungen zu dieser Konzeption machen, die häufig und zu unrecht als eine undifferenzierte Einheit aufgefaßt wird.

Die bekannteste und einflußreichste Version ist die "klassische" oder strukturelle. Sie stellt in den Spuren des politologischen Fachs "Comparative Government" einen Vergleich zwischen den totalitären Bewegungen und Regimen an, vorwiegend zwischen dem Bolschewismus bzw. Stalinismus und dem Nationalsozialismus, und arbeitet die Merkmale heraus, in denen sie übereinstimmen und durch die sie dem als Norm aufgefaßten "westlichen Verfassungsstaat " entgegengesetzt sind, etwa die bekannten sechs Merkmale von Friedrich und Brzezinski, von denen die Alleinherrschaft einer offiziellen Ideologie, die Existenz einer diktatorisch geführten Staatspartei und die Dominanz einer terroristischen Geheimpolizei die wichtigsten sein dürften. Bei Hannah Arendt fällt ein besonders starker Akzent auf den Terror und die Massenvernichtung, und die Vorgeschichte nimmt einen großen Platz ein, aber nur die Vorgeschichte des Nationalsozialismus, während der Marxismus als Vorgeschichte des Bolschewismus ausgespart wird. Weder Hannah Arendt noch Friedrich/Brzezinski setzen Bolschewismus und Nationalsozialismus umstandslos gleich, aber es sollte hervorgehoben werden, daß sie in bezug auf die Vernichtungsmaßnahmen der beiden Regime keine essentielle Unterscheidung vornehmen. Bei Hannha Arendt heißt es: „Es ist offenbar, daß die bolschewistische Propaganda ... den Mord ebenso vorbereitet wie die Nazi-Propaganda ... Die Bolschewisten lassen angeblich nur die Millionen in Arbeitslagern verrecken, die vorher bereits abgestorben waren, während die Nazis nur diejenigen in die Gaskammern schickten, die es nach den ewigen Gesetzen der Natur gar nicht hätte geben dürfen “5. Bei Friedrich und Brzezinski ist zu lesen: „Much more typical and indeed unique in its scope is the liquidation of vast masses of people, categorized in an arbitrary fashion as 'enemies of the people' and therefore unsuitable for further existence in the totalitarian system. Such was the fate of the Jews killed by the Nazis in the death camps, or of the Polish officers murdered by the Russians in Katyn." 6

Die Frage allerdings, ob nicht eine positive Kraft, eine machtvolle Überzeugung, ein in der Realität verwurzelter "Glaube“ erforderlich war, um die „Demokratie“ in großen Staaten zu vernichten, wird im Rahmen der strukturellen Version nicht ausdrücklich aufgeworfen. Sie wird von der „sozialreligiösen Ver-

${ }^{5}$ Hannah Arendt, Elemente und Ursprünge totaler Herrschaft (Frankfurt a.M. 1955, Orig.ausg. New York 1951) 550.

6 Carl J. Friedrich, Zbigniew Brzezinski, Totalitarian Dictatorship and Autocracy (Cambridge 1956) 141. 
sion" beantwortet, und es ist aufschlußreich, daß man an deren Anfang Ernst Bloch stellen darf, der sich doch weitgehend mit der einen der beiden Hauptformen des Totalitarismus identifizierte. Der Kommunismus ist für ihn nichts anderes als die endlich zu irdischen Wirkungsmöglichkeiten gelangte innerste Sehnsucht des Menschen nach dem "ganz Anderen“, nach dem "Paradies“, nach der Verwirklichung dessen, was bisher als das Subversiv-Utopische im Menschen sich nur als Protest gegen die unmenschlichen Verhältnisse und zuletzt den Kapitalismus darstellen konnte ${ }^{7}$. Der Nationalsozialismus aber ist bloß die Perversion dieser Erlösungssehnsucht, und er muß an seiner inneren Unwahrhaftigkeit zugrundegehen.

Wenige Jahre später stellte sich Erik Voegelin beiden Hauptformen des modernen Totalitarismus in gleicher Distanz gegenüber, und er stützte sich auf den negativ gefaßten Begriff der „Säkularisierung“, aber er sah sowohl im Bolschewismus wie im Nationalsozialismus einen Restbestand religiöser Glaubenskraft zu großer Stärke konzentriert, und zwar einmal in dem Enthusiasmus der Hingabe an die Idee der welterlösenden „Klasse“ und zum anderen in einer Vergötzung des „Volkes"8. Dem Sinne nach war damit auch die Frage aufgeworfen, ob nicht die westliche Moderne mit ihrer „pluralistischen Demokratie“ einerseits die elementare Voraussetzung der totalitären Sozialreligionen sei und andererseits als ein Zustand vollendeter Glaubenslosigkeit und Irreligiosität deren Telos sein könnte.

Aber erst die historisch-genetische Version der Totalitarismuskonzeption fragte ausdrücklich nach den historischen Wurzeln der totalitären Systeme und zugleich nach einem etwaigen kausalen Nexus zwischen ihnen. Als erster Begründer ist Jacob Talmon zu nennen, der 1950 im ersten Band seiner Trilogie nur die „Ursprünge der totalitären Demokratie“ bei Rousseau und anderen Autoren des 18. Jahrhunderts zum Gegenstand machte, der aber im zweiten Band schon den Konflikt zwischen der extremen Linken und einer extremen Rechten im 19. Jahrhundert schilderte, bis er 1980 im dritten Band zu der „politischen Polarisierung im 20. Jahrhundert" gelangte, wenn auch nur bis zu einem Ausblick auf die zwanziger Jahre. Karl August Wittfogel setzte 1957 in einer weitaus früheren Zeit an, nämlich bei der „hydraulischen“ oder „orientalischen“ Gesellschaft, von der her er den „voll entwickelten Apparatstaat der UdSSR“ zu verstehen suchte. Dieser reproduziere in moderner Gestalt jene „einzige herrschende Klasse, deren operativer Kern ständig als eine organisierte, zentralisierte und halbmilitärische Einheit" auftrat, so daß sie fundamental von den "herrschenden Klassen“ der „vielzentrigen und auf Privateigentum beruhenden Gesellschaft " verschieden war, in der es so etwas wie "Klassenkampf“ und damit genuine Entwicklung geben konnte. Über den Faschismus und den Nationalsozialismus sagt Wittfogel nur wenig, aber aus seiner Charakterisierung läßt sich leicht die These ableiten, daß es

7 Ernst Bloch, Erbschaft dieser Zeit (Neuausgabe Frankfurt a.M. 1962, Orig.ausg. 1935).

8 Eric Voegelin, Die politischen Religionen. Hrsg. und mit einem Nachwort versehen von Peter J. Opitz (München 1993, Orig.ausg. 1938). 
sich dabei um eine unvollkommene Gestalt des Totalitarismus gehandelt habe, die wesentliche Merkmale des westlichen und vielzentrigen Systems bewahrte, nämlich einen sozialen Pluralismus?.

Wenn die historisch-genetische Version sich in erster Linie und direkt den totalitären Phänomenen des 20. Jahrhunderts zuwenden wollte, dann mußte sie auch den Marxismus als Vorgeschichte und dessen Verwurzelung im Liberalen System und besonders in der Industriellen Revolution zum Thema machen ${ }^{10}$. Sie mußte zum zweiten das Außerordentliche und schlechthin Präzedenzlose in der bolschewistischen Revolution herausarbeiten und ernstnehmen, und zur Veranschaulichung zitiere ich bloß einen Satz des gestürzten Erich Honecker, der auf die Frage des Interviewpartners nach seiner ersten Liebe, $d$. h. nach einem Mädchen, ganz spontan antwortete: „Meine erste Liebe war eigentlich die Sowjetunion." 11 Wann je hätte es dies in der modernen Weltgeschichte gegeben, daß Hunderttausende von Deutschen, Franzosen und Italienern einen fremden Staat als die Heimstätte ihrer Überzeugungen, als geliebtes Vaterland empfanden? Dieser Enthusiasmus war jedoch nur die Kehrseite des Willens, die „Klassenfeinde“ als Feinde der Menschheit zu vernichten. Der Faschismus aber, und insbesondere dessen radikalste Gestalt, der Nationalsozialismus, ist am besten zu verstehen als die haßerfüllte Antwort auf diesen, wie er es empfand, Verrat am wirklichen Vaterland und diese Selbstidentifizierung mit einem "asiatischen“ $P$ Pänomen ${ }^{12}$. Als radikale Antwort mußte er indessen die gleiche Anschaulichkeit der Feindbestimmung und ein ähnliches Überschreiten der nationalen Grenzen anstreben, und daher wurde die Ersetzung „des Kapitalisten“ durch „den Juden“ und die Verdrängung der Nation durch die „Rasse“ notwendig. Der ursprünglichen Erlösungs- und Vernichtungsideologie trat eine postulatorische und artifizielle, aber ebenfalls kraftvolle Erlösungs- und Vernichtungsideologie gegenüber, abkürzend und etwas plakativ ausgedrückt: der Gulag war ursprünglicher als Auschwitz, aber das eine unterschied sich gleichwohl wesentlich von dem anderen, und beide müssen als Bestandteile des „europäischen Bürgerkrieges von 1917 bis 1945“ gesehen werden $^{13}$. Jener Sieg der pluralistischen Demokratie in der „Wende“ der Jahre 1989/91

${ }^{9}$ Karl August Wittfogel, Die orientalische Despotie. Eine vergleichende Untersuchung totaler Macht (Köln, Berlin 1962, Orig.ausg. 1957) 452, 410, 552.

10 Ernst Nolte, Marxismus und Industrielle Revolution (Stuttgart 1983).

11 Reinhold Andert, Wolfgang Herzberg, Der Sturz. Erich Honecker im Kreuzverhör (Berlin, Weimar 1990) 129.

12 Ernst Nolte, Der Faschismus in seiner Epoche (München 1963) bes. 51.

13 Ernst Nolte, Der europäische Bürgerkrieg 1917-1945. Nationalsozialismus und Bolschewismus (Frankfurt a.M., Berlin 1987).

Der Begriff „europäischer Bürgerkrieg“ ist nicht von mir erfunden worden. In aller Regel wird er aber als Metapher für die europäischen Staatenkriege des 20. Jahrhunderts verstanden, die als Bürgerkriege innerhalb der übergeordneten Einheit Europas verstanden werden. Donald C. Watt setzt in seinem Artikel "The European Civil War" (in: Wolfang J. Mommsen, Lotbar Kettenacker, The Fascist Challenge and the Policy of Appeasement (London 1983) 3- 
nimmt, von hier aus betrachtet, ein Aussehen an, das die Verschiedenheit der beiden wichtigsten "Antikommunismen" herausstellt, aber ihre Ähnlichkeit nicht leugnet und das von einer bloßen Selbstverherrlichung des "westlichen Systems" weit entfernt ist. Hätte man sich klargemacht, daß der Versuch einer Gesamtinterpretation des 20. Jahrhunderts vorlag, der zugleich eine Synthese des Denkens bedeutender Vorgänger war - und nicht etwa eine "Faschismustheorie“ oder ein "Revisionismus", - wäre im sogenannten Historikerstreit weniger an Aufregung und mehr an Nachdenken zu verzeichnen gewesen.

21) den Begriff in Gegensatz sowohl zu dem Verständnis der "großen Mächte“ wie zu der Konzeption eines „Manichean Struggle between Competing Ideologies“. 
